\title{
VICTORIAN CONTINGENCIES
}


This page intentionally left blank 


\section{Victorian \\ Contingencies}

Experiments in Literature, Science, and Play

TINA YOUNG CHOI 
(C)2022 by Tina Young Choi. All rights reserved.

No part of this book may be reproduced or transmitted in any form or by any means, electronic or mechanical, including photocopying and recording, or in any information storage or retrieval system without the prior written permission of Stanford University Press.

Printed in the United States of America on acid-free, archival-quality paper

Library of Congress Cataloging-in-Publication Data

Names: Choi, Tina Young, author.

Title: Victorian contingencies : experiments in literature, science, and play / Tina Young Choi.

Description: Stanford, California : Stanford University Press, [2022] | Includes bibliographical references and index.

Identifiers: LCCN 2021007876 (print) | LCCN 2021007877 (ebook) | ISBN 9781503629288 (cloth) | ISBN 9781503629769 (ebook)

Subjects: LCSH: English literature—19th century-History and criticism. | Science-

Great Britain—History—19th century. | Amusements—Great Britain - History-

19th century. | Great Britain—Civilization—19th century. | Great Britain—Social life and customs - 19th century.

Classification: LCC DA533 .C545 2022 (print) | LCC DA533 (ebook) | DDC 941.081—dc23

$\mathrm{LC}$ record available at https://lccn.loc.gov/2021007876

LC ebook record available at https://lccn.loc.gov/2021007877

Cover illustration: Two views of Mt. Vesuvius, interleaved, from "Spooner's Protean Views"

(London: W. Spooner, c. 1840).

Cover design: George Kirkpatrick

Text design: Kevin Barrett Kane

Typeset at Stanford University Press in 11/15 Arno Pro 